\title{
Elipse como lugar geométrico en un ambiente de geometría dinámico
}

\section{Ellipse as Locus of Points in a Dynamic Geometry Environment}

José Carlos León
Universidad de Lima

\section{RESUMEN}

Presentamos los resultados de una investigación que tuvo como objetivo potenciar en los estudiantes el desarrollo del pensamiento dinámico en torno al carácter variacional de los saberes matemáticos. Planteamos situaciones que involucran cambios y patrones de regularidad en las magnitudes estudiadas cuando transcurren de un estado a otro, y cuyos tratamientos demandan por parte de los sujetos, procesos cognitivos que permitan explicar, conjeturar, argumentar, compartir y modelar significados de los objetos tratados. Mostraremos un episodio correspondiente a la condición geométrica de la elipse para alumnos de los cursos de precálculo en un ambiente de geometría dinámica, haciendo uso del GeoGebra como instrumento mediador. Para mostrar el uso de diversas representaciones empleamos como marco de referencia, el Registro de Representación Semiótica, el cual analiza el rol que cumplen los signos como registros representativos de los objetos matemáticos.

\section{Palabras clave:}

Registros de representación semiótica / Pensamiento y lenguaje variacional / GeoGebra

\section{ABSTRACT}

In this document we present the results of a research that aimed to enhance in the students' the development of dynamic thinking about the variational nature of mathematical knowledge. We presented situations involving changes and regular patterns in the quantities studied when passing from one state to another, and whose study required students to develop cognitive processes that explain, speculate, argue, share and model the meaning of the objects under analysis. We will present the case of the geometric condition of the ellipse for students in pre-calculus courses in an environment of dynamic geometry using GeoGebra as a mediator instrument. To demonstrate the use of different representations we use as a reference, the Semiotics Representation Register which analyzes the role of signs as representative records of mathematical objects.

\section{Keywords:}

Semiotics Representation Register / Variational Thinking and Language / GeoGebra 
E n esta publicación mostramos un episodio que tiene por objetivo potenciar el desarrollo del pensamiento y lenguaje dinámico de la matemática. Propiciamos la puesta en juego de diversas estrategias para generar argumentos que permitan el surgimiento y el descubrimiento progresivo de la condición geométrica de la elipse, identificando las magnitudes involucradas en los cambios y reconociendo ciertos patrones de regularidad, con la intención de estimar y explicar algunos resultados del fenómeno de variación.

Esta mirada de las matemáticas hacia el desarrollo del Pensamiento y lenguaje variacional (Pylvar) permitió vincular el significado gráfico con la representación numérica de la condición geométrica de la elipse, lo cual demandó, por parte del alumno, el desarrollo de descripciones cualitativas, articuladas por expresiones verbales, gestuales, las cuales fueron cuantificadas por secuencias numéricas, tablas o representaciones gráficas que fueron desarrolladas en ambientes de geometría dinámica. De allí que surgió la necesidad de reconocer que existen otros planteamientos con sus propias complejidades que abarcan representaciones y construcciones geométricas, las cuales sirven como una propuesta adicional al proceso de enseñanza y aprendizaje de la geometría analítica actual, específicamente la elipse, donde, tradicionalmente, ha prevalecido el enfoque algebraico

Respecto al Pylvar, coincidimos con los autores Caballero y Cantoral (2013), cuando señalan que se hace necesario propiciar en los alumnos acciones asociadas a la comparación de dos o más estados, con el fin de que encuentren diferencias o similitudes entre ellos, y a partir de dichos comportamientos estimar y predecir, para fomentar un aprendizaje rico en significados. De igual forma, cuando enfatizan que muchas veces el discurso matemático que se da en la clase está enfocado en procedimientos y estrategias memorísticas que, aunque necesarios, no permiten una construcción de la naturaleza de los significados.

Algunos de los estudios realizados por Vasco (2006), dan cuenta que incluso las representaciones gestuales son, en algunos casos, más efectivas que cualquier fórmula ya que representan demostraciones cualitativas de variación para indicar el aumento o disminución de lo que cambia. El autor añade que los mejores problemas deberían ser desafíos o retos que impliquen la modelación de algún proceso y no propiamente la tradicional resolución de problemas y ejercicios. Dicho pensamiento está en contraposición con las fórmulas entendidas sólo para el reemplazo de valores numéricos que no enfatizan qué varía y con respecto a qué varía.

Por tal motivo, el autor afirma vivenciar, ejercitar los procesos de matematización y modelación matemática desde el preescolar y la primaria, configurando los currículos, programas, unidades didácticas, textos, materiales y juegos matemáticos. Añade que este tipo de pensar de manera dinámica se desarrolla de múltiples maneras. Indica que el pensamiento variacional se desarrolla con el pensamiento numérico y aquellos intentos por captar patrones de progresión. También ocurre con el pensamiento geométrico, por ejemplo, en la variación del área de un triángulo, si uno de los vértices experimenta un cambio de posición a lo largo de una paralela a la base o cuando la base se modifica a lo largo de la recta mientras se mantiene el vértice fijo. Vasco (2006) también señala que dicho pensamiento se desarrolla con el pensamiento proporcional siempre que se busque la covariación entre magnitudes proporcionales y nos alejemos de la definición tradicional que la señala como una igualdad de dos razones. Con representaciones gráficas y tabulares, pero con el respaldo de algún programa de geometría dinámica. Con representaciones gestuales que permitan expresar, con nuestro cuerpo o expresiones, cómo las magnitudes involucradas cambian. En fin, de acuerdo a las investigaciones referidas, son las prácticas relativas a la variación, aquellas que simulan el dinamismo de los procesos o fenómenos reales, las que favorecen la construcción de un concepto determinado.

Por tal motivo, y en relación al pensamiento variacional, mostramos en la figura 1, un deslizador $\Delta \mathrm{x}$ un punto $P$ sobre el eje de abscisas y la función representada por una línea poligonal. El triángulo sombreado, que se muestra en la figura 2, obedece al uso de una herramienta que se creó y que aparece al final de la barra de herramientas. El comando que se aplicó para dicha herramienta obedece a la expresión $(\mathrm{x}(\mathrm{P})+\Delta \mathrm{x}, 0)$.

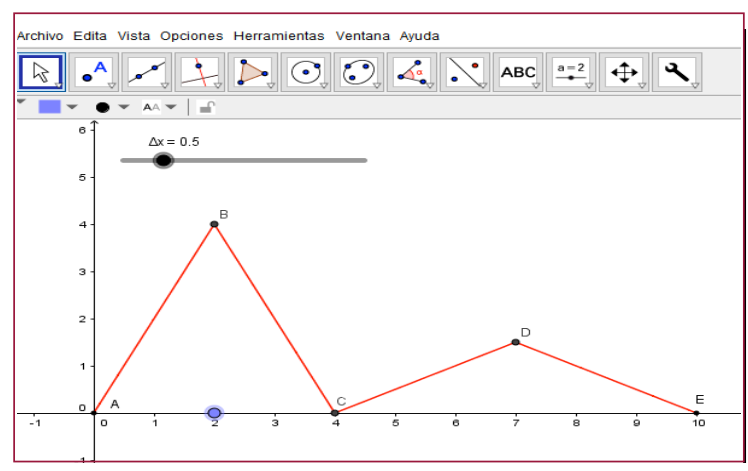

Figura 1. Deslizador, punto y línea

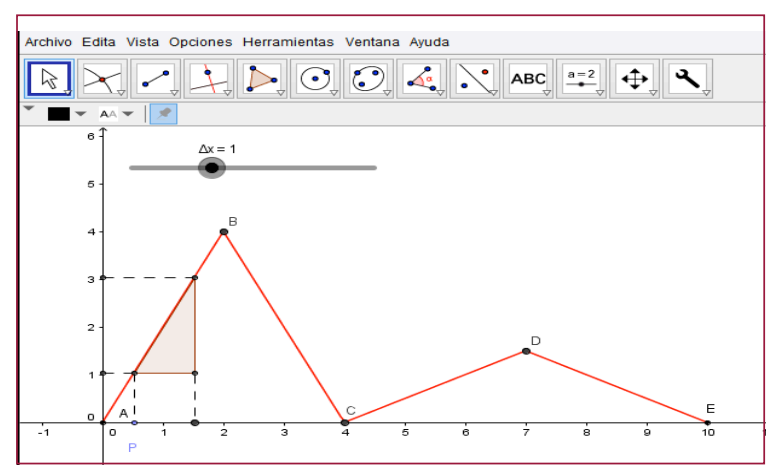

Figura 2. Triángulo dinámico. 
Para movilizar la herramienta creada, se hace un clicken un punto $P$ sobre el eje de abscisas, otro en el deslizador $\Delta x$ y un tercero en la línea poligonal mostrada; entonces otro punto aparecerá sobre el eje de abscisas, alejado $\Delta x$ unidades del punto $P$ y acompañado por un triángulo tal como se muestra en la gráfica de la figura 2. Estas construcciones permiten modificar el cateto horizontal del triángulo, haciendo uso del deslizador $\Delta x$, y trasladar el triángulo a lo largo de la línea poligonal.

Si cambia el valor del deslizador $\Delta x$, automáticamente cambian los catetos del triángulo y propiciamos que el sujeto perciba visualmente dichas variaciones. Si el triángulo se desplaza sobre el mismo segmento de recta, el alumno identifica que $\Delta y$ mantiene el mismo patrón de regularidad. Cuando la recta cambia de inclinación, por ejemplo, si el triángulo transita del segmento $A B$ al segmento $C D$, se origina una variación en el cateto vertical del triángulo que corresponde a la variable $\Delta y$.

Además, se observa que $\Delta y$ pueden llegar a valores cercanos a cero, en la medida en que la recta se vaya posicionando horizontalmente. Las gesticulaciones, murmuraciones, ademanes, son algunos de los comportamientos que los sujetos expresan como un intento de reproducir cualitativamente los cambios que se perciben en diversos puntos de los tramos de la gráfica mostrada.

Se pueden cuantificar dichas observaciones tomando como referencias algunos puntos de análisis donde aparezcan cambios. En las tablas 1 y 2, el alumno escribe en los espacios correspondientes las variaciones de las ordenadas cuando el de las abscisas $\Delta x$ permanece constante e igual a 1.

\begin{tabular}{|l|c|l|}
\hline \multirow{2}{*}{ Intervalo } & \multicolumn{2}{|c|}{ Estudio del comportamiento } \\
\cline { 2 - 3 } & Cambio abscisas & Cambio ordenadas \\
\hline $0 \leq x \leq 1$ & 1 & \\
\hline $0.5 \leq x \leq 1.5$ & 1 & \\
\hline $1 \leq x \leq 2$ & 1 & \\
\hline
\end{tabular}

Tabla 1. Valor de la pendiente tramo AB.

\begin{tabular}{|l|c|l|}
\hline \multirow{2}{*}{ Intervalo } & \multicolumn{2}{|c|}{ Estudio del comportamiento } \\
\cline { 2 - 3 } & Cambio abscisas & Cambio ordenadas \\
\hline $4 \leq x \leq 5$ & 1 & \\
\hline $5 \leq x \leq 6$ & 1 & \\
\hline $6 \leq x \leq 7$ & 1 & \\
\hline
\end{tabular}

Tabla 2. Valor de la pendiente tramo CD.

De acuerdo a las preguntas que se formulen, el alumno moviliza la estrategia de comparación para establecer diferencias entre dos estados. Estima que el cambio de la ordenada se mantiene constante en el tramo $A B$ y disminuye para el tramo $C D$ cuando el segmento de recta se inclina hacia el eje de las abscisas en el sentido horario.

En dicho registro de representación numérica, los alumnos identifican a las variaciones de la ordenada como positivas de acuerdo a la relación $\Delta \mathrm{y}=f(\mathrm{x}+\Delta \mathrm{x})-f(\mathrm{x})>0$, que es parte de su saber previo, y a la que recurren como un instrumento de conocimiento matemático, específico en el planteamiento de sus estrategias variacionales.

De manera análoga, mostramos en las figuras 3 y 4 el estudio de una representación dinámica cuando el triángulo que se desliza por los tramos $\boldsymbol{B C}$ y $\boldsymbol{D E}$.

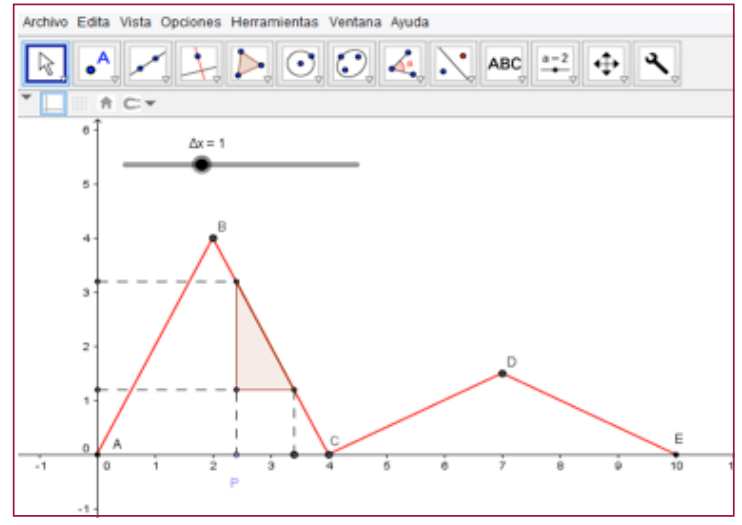

Figura 3. Pendiente segmento BC.

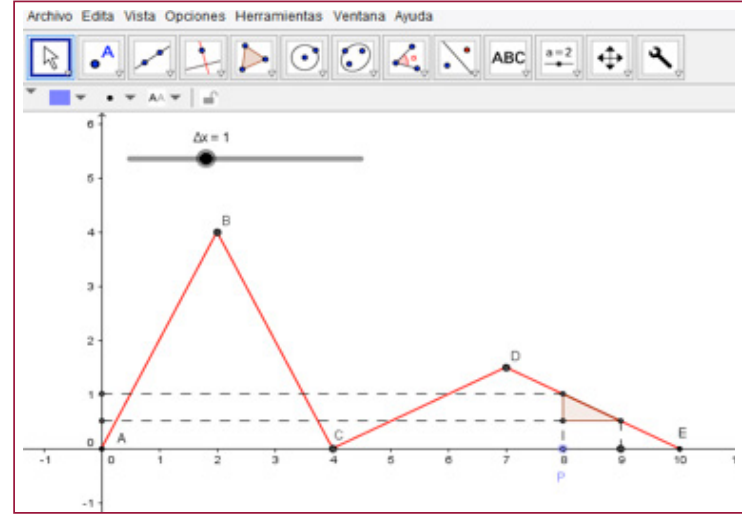

Figura 4. Pendiente segmento DE. 
Las comparaciones de los tramos mencionados y que son mostrados en la figura, nos indican que el cambio de la ordenada $\Delta \mathrm{y}=f(\mathrm{x}+\Delta \mathrm{x})-f(\mathrm{x})<0$ es negativo y permanece constante en cada tramo, aunque dicho valor se hace menos negativo 0 se acerca a cero a medida que el segmento de recta se inclina a la izquierda.

En suma, la matemática nos brinda la oportunidad de propiciar tareas en relación al pensamiento dinámico matemático, que permitan, en un primer momento, la captación de lo que cambia o lo que queda constante, y, luego, la mediación de sus conocimientos que articulen las estrategias correspondientes para explicar, modelar y cuantificar los retos que plantea la situación variacional. Podemos hallar diversos contextos en los que podemos incidir en este tipo de pensamiento variacional, como ocurre con los cambios entre los lados de un triángulo rectángulo por la causalidad del ángulo de elevación, con el desplazamiento lineal de un móvil en un tiempo determinado, con la trayectoria de los puntos sobre superficies cónicas las cuales corresponden a lugares geométricos, con las razones de cambio, entre otros.

Todas ellas, de acuerdo a lo que indica Vasco (2006), permiten la producción de sistemas o modelos mentales que, gracias a procedimientos matemáticos permiten abordar los estados de cambio, logrando explicar el modelo mental, y luego comparando los resultados en el proceso que se quiere modelar.

Al respecto, el autor plantea un acercamiento al concepto del pensamiento variacional:

"El pensamiento variacional puede describirse aproximadamente como una manera de pensar dinámica, que intenta producir mentalmente sistemas que relacionen sus variables internas de tal manera que covaríen en forma semejante a los patrones de covariación de cantidades de la misma o distintas magnitudes en los subprocesos recortados de la realidad." (Vasco, 2006, p. 139).

De acuerdo a esta mirada, a partir de ciertos tipos de procesos o sistemas, nuestros cerebros puedan estudiar, construir 0 recrear un modelo simplificado. En dicho modelo debe contemplarse aquellos componentes que comparten una red de relaciones específicas y cuyas estimaciones y predicciones hagan posible explicar la realidad. Creemos que los actuales ambientes de geometría dinámica pueden sintonizar directamente con la recreación de dichos recortes de la realidad y simular los patrones de regularidad que se requiere mostrar.

Esta forma de hacer matemática, representa, según Vasco (2012), el quehacer de la matemática, la cual es construir, manipular, estudiar, refinar, admirar y disfrutar los modelos mentales simplificados que nuestro cerebro puede crear a partir de cualquier tipo de proceso. Este planteamiento apoya la tesis que señala que la aplicación de un algoritmo o solo la verificación del cumplimiento de alguna regla, no implica la comprensión de los procesos de variación. Esto significa que urge el empleo de un conjunto de elementos o características que conformen el desarrollo del pensamiento y lenguaje variacional.

Así, destacamos a Caballero y Cantoral (2013), que dan cuenta de la evidencia del desarrollo del pensamiento variacional y describen un modelo de interacción de los elementos del Pylvar orientados a potenciar el desarrollo del pensamiento dinámico en el alumno.

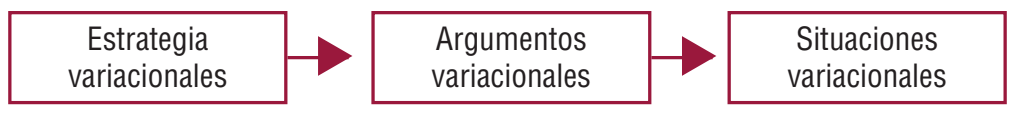

Figura 5. Interacción de los elementos base del Pylvar. Elaboración propia.

En la figura 5 mostramos algunos de los elementos que el autor, indica, caracterizan el Pylvar. En dichas vivencias, los sujetos ponen en juego una serie de estrategias que le sirven para abordar o enfrentar la situación o fenómeno y que implica el reconocimiento del cambio o del patrón de regularidad de las magnitudes involucradas en la situación dada. Además, el autor precisa que la estrategia variacional implica recurrir a la comparación de dos o más estados sucesivos para hallar un patrón de comportamiento, de tal forma que pueda estimar y predecir otros estados para anticipar uno nuevo, como modelo predictivo. En esa transición, una predicción global encierra un cambio general de las predicciones, cuánto crece 0 disminuye, y una predicción local, el valor numérico de ese cambio.

Finalmente, del resultado de las estrategias o prácticas variacionales, se generan los argumentos que son articulados por códigos variacionales, como frases, dibujos, esquemas o gráficos, con los cuales los sujetos tienen posibilidad de interactuar para explicar las situaciones variacionales propuestas. A continuación, mostramos el marco teórico que fundamenta la investigación de la experiencia de enseñanza de aprendizaje. 


\section{Marco teórico}

En este artículo mostramos cómo los conceptos de la matemática requieren el uso de más de un sistema de representación y de la coordinación de dichas representaciones para alcanzar la comprensión de algunas de sus propiedades. Para ello, hacemos referencia a las investigaciones realizadas por Duval (2006) en el campo de los registros de representación, con el cual los objetos matemáticos y sus correspondientes actividades cognitivas del pensamiento, necesitan de una serie de representaciones constituidas por el empleo de signos con los cuales es posible explorar los significados 0 creencias que un sujeto pueda tener de dicho objeto.

De acuerdo a la Teoría de Registro de Representaciones Semiótica, no es posible acceder a los objetos matemáticos, los cuales no son accesibles por la percepción ni manipulables como un objeto físico, fuera de un sistema semiótico. En ese sentido, indica que representaciones semióticas como las gráficas, expresiones algebraicas, numéricas, figuras geométricas y enunciados, son relevantes en la medida en que sean formados haciendo uso de un conjunto de signos identificables del propio sistema de formación, tratados con reglas inherentes al mismo registro de representación para que sean transformados y convertidos en un registro distinto de representación, pudiendo conservar la totalidad o una parte de la representación inicial.

Entre los aspectos más importantes a dicho marco teórico destacamos la comprensión integral de un objeto cuando está basado en la coordinación de al menos dos sistemas de representación pertenecientes a registros diferentes. En el artículo que presentamos, recurrimos al dinamismo de los registros gráficos, algebraicos y numéricos, haciendo uso de un programa de ambiente de geometría dinámica, con actividades que exigen el desarrollo de las operaciones cognitivas tratamiento y conversión de los registros correspondientes.

Creemos, al igual que el autor, que la pluralidad de los registros de representación conduce a caminos diversos por donde el sujeto pueda transitar en función de las singularidades de su aprendizaje.

\section{Análisis de la experiencia desarrollada}

Se muestra el análisis de una actividad mediada por el GeoGebra, la cual está orientada a propiciar la noción de la condición geométrica de la elipse mediante una secuencia de tareas centradas en focalizar el desarrollo del pensamiento y lenguaje variacional. Los participantes usaron como estrategia variacional la comparación de estados sucesivos 0 seriación para identificar covaraciones y establecer un modelo que cuantificara el proceso dinámico de construcción. Se logró, además, la conversión del objeto a un registro distinto de representación de acuerdo a la Teoría de Registro de Representaciones Semiótica.

En la figura 6 se muestra el gráfico de un archivo creado en GeoGebra. Se observa una circunferencia de centro $C$ un punto $P$ que puede recorrer la circunferencia en sentido antihorario, haciendo uso del deslizador $\alpha$ y un punto $I$, perteneciente a la región de la circunferencia.

Como primera tarea, los participantes deben determinar un punto $Q$ sobre el radio $C P$ que equidiste de los puntos $P$ e $I$, justificando su procedimiento.
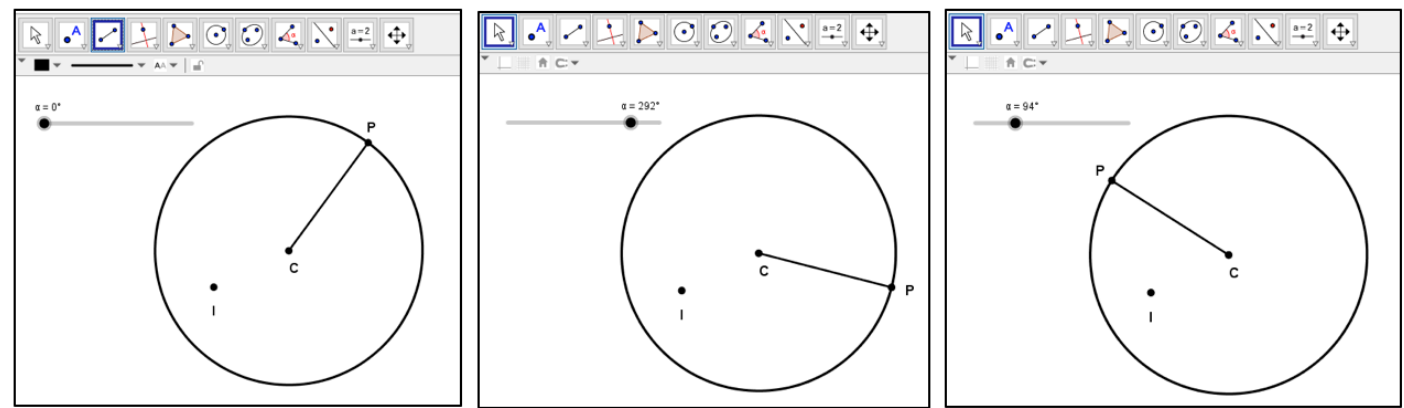

Figura 6. Desplazamiento del punto P sobre la circunferencia. 
En la primera ilustración de la figura 7 se observan las acciones que dieron lugar a la determinación del punto $Q$. Los participantes trazan la mediatriz del segmento $\boldsymbol{P I}$ y determinan, en la intersección con el radio, el punto solicitado. Sus acciones son justificadas con la propiedad que indica que todo punto perteneciente a dicha recta equidista de las extremidades del segmento.
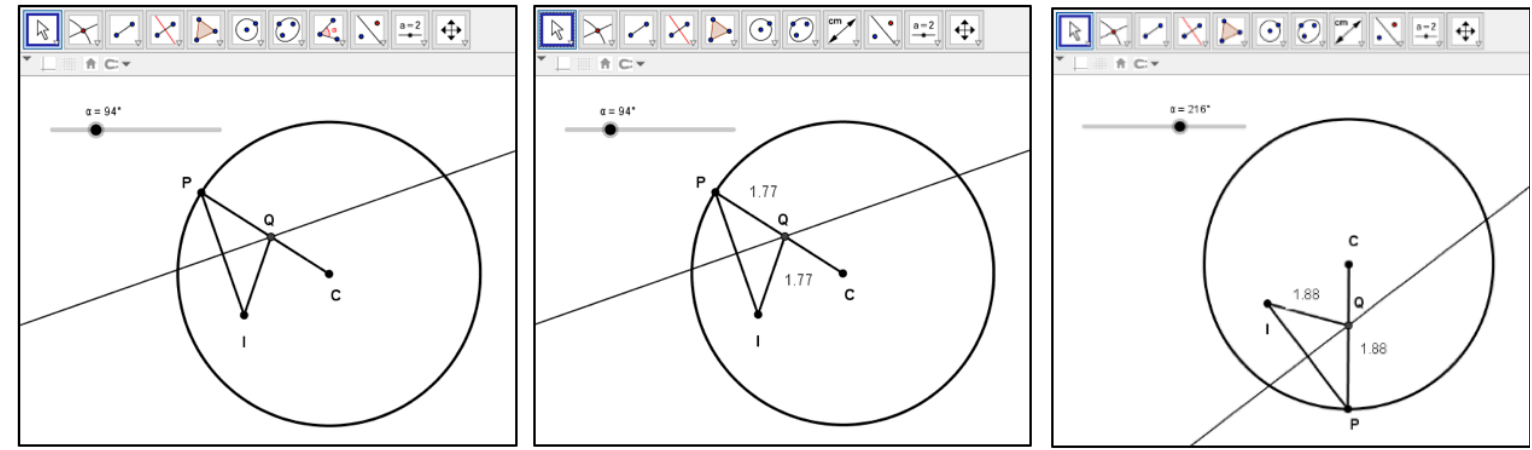

Figura 7. Trazo de la mediatriz para la determinación del punto Q.

La segunda y tercera ilustración de la figura 7 es en respuesta al requerimiento de la segunda tarea sobre las magnitudes que conservan un patrón de regularidad en torno al sistema creado. Los participantes hacen uso de la herramienta Distancia para visualizar las longitudes de los segmentos $Q P$ y $Q$ l, y determinar que siempre se mantiene la equivalencia entre dichos segmentos. De igual forma establecen que el triángulo isósceles y el radio de la circunferencia mantienen propiedades de invariabilidad.

En la siguiente tarea se les pide trazar, con la herramienta polígono, el triángulo QIC, y se les pregunta acerca del reconocimiento de algunas propiedades invariantes en dicho triángulo: ¿qué sucede con el perímetro del triángulo QIC cuando se hace uso del deslizador a?, ¿se mantiene constante 0 varía?, ¿qué argumentos validan su respuesta a las preguntas planteadas?

Conforme se observa en la primera ilustración de la figura 8, esta situación posibilita a los participantes establecer características cualitativas y cuantitativas de la situación, y hacer uso de la estrategia de seriación, pues es posible el análisis de los estados sucesivos cuando mueve el deslizador, estableciendo, de este modo, relaciones entre los segmentos.
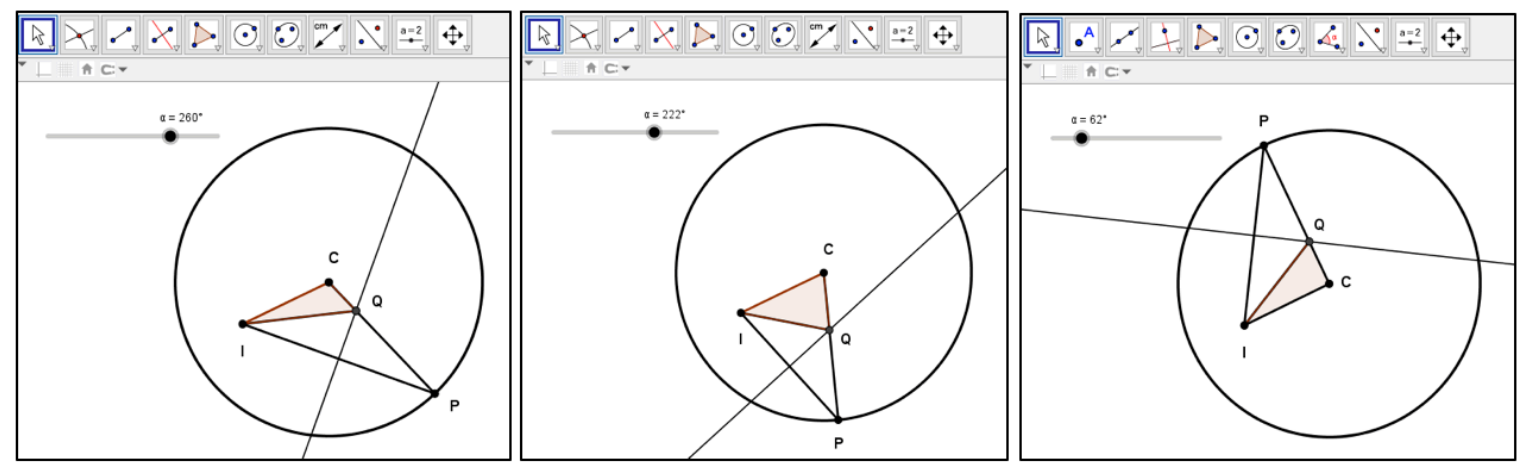

Figura 8. Construcción de triángulos de igual perímetro.

Los estudiantes no mostraron ninguna dificultad para señalar que la longitud de la base $C /$ del triángulo $C Q /$ siempre es la misma y que la suma de las longitudes de los segmentos QI y QC es una constante igual a la longitud del radio de la circunferencia.

La figura 9 da cuenta del triángulo CQ/ de perímetro constante. 


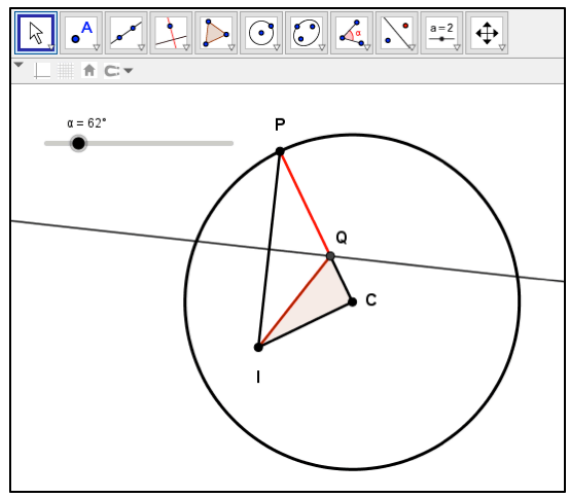

Figura 9. Triángulo de perímetro constante.

Además, en el triángulo el punto $Q$ es el único que describe un lugar geométrico cuando es movilizado por el deslizador, pues el centro y el punto interior permanecen fijos. Esto conduce a la siguiente tarea, en la que los alumnos visualizan el lugar geométrico haciendo uso de la herramienta Rastro.
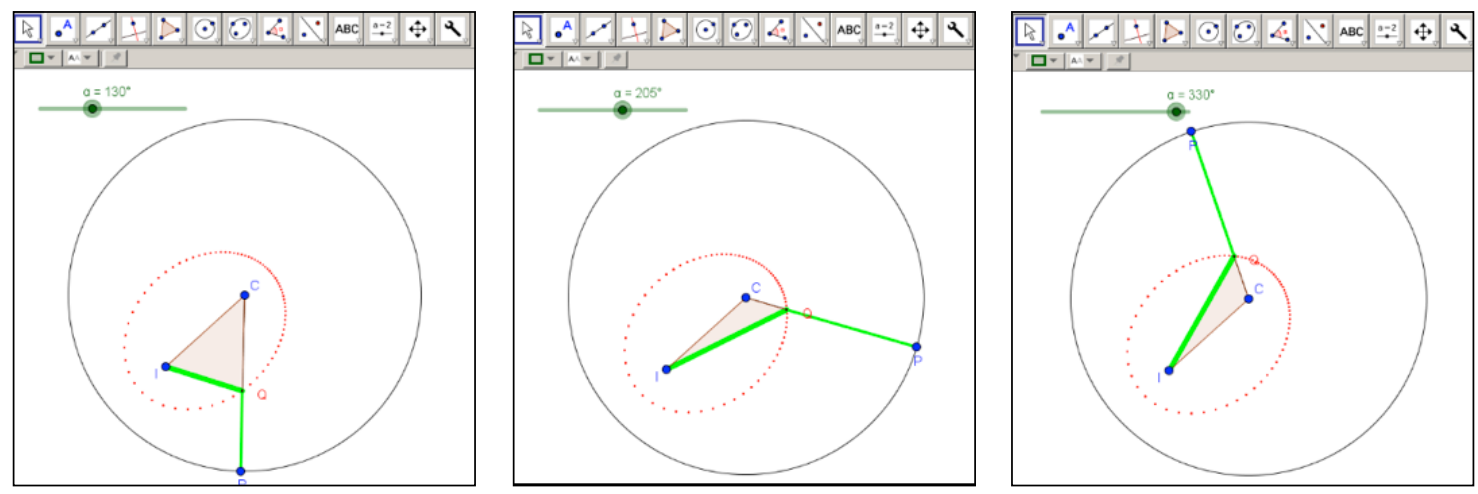

Figura 10. Lugar geométrico de la elipse.

La figura 10 muestra cómo los alumnos vincularon la trayectoria del punto Q a la gráfica de una elipse y escriben su condición geométrica como la suma de las medidas de los segmentos QC y Q/ igual a una constante equivalente al radio de una circunferencia con centro en $C$ y un punto fijo / en su interior.

Esta actividad me permite crear un puente entre la geometría sintética con la geometría analítica, teniendo en cuenta al GeoGebra, que actúa como agente mediador, pero integrador en el conocimiento matemático, facilitando las representaciones semióticas como las gráficas, expresiones algebraicas y numéricas, como se muestra en la figura 11.

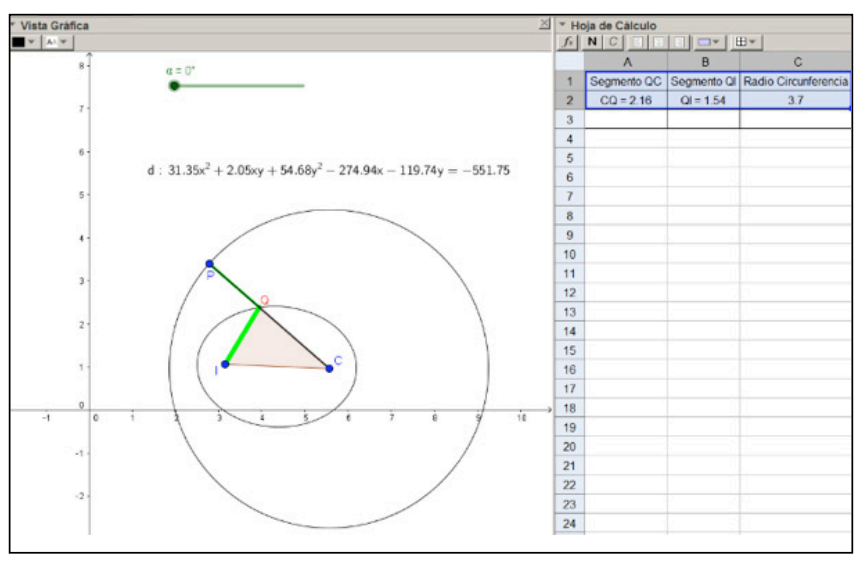

Figura 11. Registros representación de la elipse. 
De acuerdo a la teoría de Duval (2006), las figuras geométricas fueron formadas y tratadas con reglas propias del sistema de representación. Además, gracias a la Vista Hoja de Cálculo y Vista Algebraica del GeoGebra, estas fueron convertidas a otro registro distinto de representación, en este caso, el registro numérico y algebraico.

Es importante resaltar que en nuestro tratamiento hemos dado menor trascendencia a las expresiones algebraicas pero mayor énfasis a las construcciones en torno al pensamiento variacional. De allí que, además del desarrollo del Pylvar, hemos tendido un puente que vincula los procedimientos algebraicos y las construcciones geométricas.

\section{Reflexiones}

Creemos fundamental potenciar el desarrollo del pensamiento dinámico en la matemática, haciendo uso de programas interactivos como el GeoGebra, los cuales nos permiten recrear, simular o modelar de manera simplificada procesos de la realidad y cuyas estimaciones o predicciones a partir de dichos modelos hacen posible explicar, manipular o modificar dichos procesos.

La integración de un programa de geometría dinámica a nuestros quehaceres matemáticos ofrece un ambiente de enseñanza y aprendizaje que nos posibilita acercarnos a la producción del conocimiento enfatizando la recreación de ambientes dinámicos.

Esta propuesta busca evidenciar aspectos del pensamiento variacional en los alumnos cuando movilizan estrategias en la determinación de la condición geométrica de la elipse como lugar geométrico. Nos alejamos de los procesos tradicionales en la que se incluyen procesos algebraicos y nos acercamos, en todo caso, a crear un vínculo entre los registros gráficos y algebraicos.

La interacción entre el programa GeoGebra y el Pylvar permitió el surgimiento de otras construcciones básicas, como representaciones algebraicas y registros numéricos, los cuales fueron abordados desde el contexto dinámico de la matemática.

\section{Bibliografía}

Caballero, P. y Cantoral, R. (2013). "Una caracterización de los elementos del pensamiento y lenguaje variacional”, en Flores, R. (ed.). Aspectos socioepistemológicos en el análisis y el rediseño del discurso matemático escolar. (Acta Latinoamericana de Matematica Educativa ALME 26, pp. 1195-1203). México: Comité Latinoamericano de Matemática Educativa.

Duval, R. (2006). "Un tema crucial en la educación matemática: La habilidad para cambiar el registro de representación”, en La Gaceta de la Real Sociedad Matemática Española (Madrid), vol. 9, núm. 1, RESME, pp. 143-168, trad. Humberto Quesada. Madrid: Universidad Complutense, Facultad de Matemáticas.

VAsco, C. (2006). "El pensamiento variacional, la modelación y las nuevas tecnologías”, en Vasco, C. (ed.). Didáctica de las matemáticas: Artículos selectos. Bogotá: Universidad Pedagógica Nacional, pp.134-148.

Vasco, C. (2012) "Experiencia interna y quehacer matemático", en CADE (ed.). Perspectivas en la didáctica de la matemática. Bogotá: Doctorado Interinstitucional en Educación Universidad Distrital Francisco José de Caldas, pp. 111-136. 